\title{
Polymyxin B-Immobilized Fiber Column Direct Hemoperfusion for Micro-Preemie Infants: Is Extended Duration Better than Early Start?
}

\author{
Naoto Nishizaki ${ }^{a}$ Kaoru Obinata ${ }^{a}$ Toshiaki Shimizu $^{b}$ \\ aDepartment of Pediatrics, Juntendo University Urayasu Hospital, Chiba, Japan; ${ }^{b}$ Department of Pediatrics, \\ Juntendo University Faculty of Medicine, Tokyo, Japan
}

Dear Editor,

As we perused the article "Impact of Extended Duration of Polymyxin B-Immobilized Fiber Column Direct Hemoperfusion on Hemodynamics, Vasoactive Substance Requirement, and Pulmonary Oxygenation in Patients with Sepsis: An Observational Study" by Mitaka et al. [1] with great interest, we deemed it necessary to reappraise their viewpoints regarding polymyxin $\mathrm{B}$-immobilized fiber column direct hemoperfusion (PMX-DHP) strategies for patients with septic shock. According to their retrospective study of 103 adult patients with sepsis who underwent PMX-DHP, the time required to start PMX-DHP was not associated with significant differences in 28-day mortality between the 2 groups. While we partly concur with their conclusion, the evidence of the efficacy and safety of PMX-DHP for neonates is limited due to the lack of clinical trials. Thus, strategies for neonates, especially micro-preemie infants, need to be considered separately from the adult findings.

Since 2011, a PMX-DHP column specialized for neonates with a priming volume of only $8 \mathrm{~mL}$ (Toraymyxin ${ }^{\circledR}$ 01-R; Toray Medical Co., Tokyo, Japan) has been available in Japanese clinical settings. We have been using PMX-DHP for neonatal septic shock and have published several of our experiences [2-6]. Similar to the successful experience described by Mitaka et al. [1] , we use PMX-

karger@karger.com

(c) 2021 S. Karger AG, Basel

www.karger.com/bpu

Karger!
DHP as long as possible, instead of the conventional duration (2 $\mathrm{h}$ per session [7]) when there are no adverse events and extracorporeal circulation is possible. As seen in our results (shown in Table 1), the shorter the time from the decision to initiate PMX-DHP, the lower the 28day mortality and hospital mortality. With neonates, especially micro-preemie infants weighing around $1,000 \mathrm{~g}$, it is often difficult to ensure blood access to withstand extracorporeal circulation. Specifically, it takes time to place an umbilical arteriovenous catheter immediately after birth or to secure large blood vessels such as the median arteriovenous. In addition, it often takes longer to start PMX-DHP due to a more unstable hemodynamic condition in neonates than in adult patients. Based on our experience, we confirm that even if PMX-DHP is performed for $2 \mathrm{~h}$ or longer, prognosis tends to deteriorate in cases where it takes a longer time to initiate PMX-DHP.

In connection with the aforementioned aspects, we have previously reported interesting clinical findings in neonates [4]. Early-onset sepsis (EOS) occurring during the first $72 \mathrm{~h}$ of life had a better prognosis than late-onset sepsis (LOS) occurring after $72 \mathrm{~h}$ of life. On comparing the 2 groups ( $E O S=6$ vs. $L O S=5)$, the 28 -day mortality rate after admission to the neonatal intensive care unit and hospital mortality were significantly lower in the EOS group than those in the LOS group ( $0 \%$ vs. $60 \%, p=0.026$; 
Table 1. Patient characteristics, implementation of PMX-DHP, and mortality

\begin{tabular}{|c|c|c|c|c|}
\hline Parameter & $\begin{array}{l}\text { All patients* } \\
(n=9)\end{array}$ & $\begin{array}{l}\text { appropriate group } \\
(n=5)\end{array}$ & $\begin{array}{l}\text { delayed group } \\
(n=4)\end{array}$ & $p$ value \\
\hline Gestational age, wk & $26(25.0,28.0)$ & $28(27.5,30.8)$ & $24(24.3,25.8)$ & 0.85 \\
\hline Birth weight, $g$ & $845(689,919)$ & $845(788,867)$ & $760(537,1,361)$ & 0.66 \\
\hline Male, $n(\%)$ & $6(67)$ & $2(40)$ & $4(100)$ & 0.12 \\
\hline Total duration of PMX-DHP, $\mathrm{h}$ & $5.4(5.2,6.5)$ & $6.0(5.2,6.5)$ & $5.3(5.0,5.4)$ & 0.56 \\
\hline Time for decision to induction of PMX-DHP, min & $70(60,120)$ & $60(55,65)$ & $138(120,155)$ & 0.02 \\
\hline 28-d mortality, $n(\%)$ & $2(22)$ & $0(0)$ & $2(50)$ & 0.16 \\
\hline Hospital mortality, $n$ (\%) & $3(33)$ & $0(0)$ & $3(75)$ & 0.047 \\
\hline
\end{tabular}

The qualitative data are shown as $n(\%)$ and the quantitative data are shown as medians (interquartile range). Appropriate means within 70 min from the decision to implement PMX-DHP to the start. Delayed means after 70 min from the decision to implement PMX-DHP to the start. PMX-DHP, polymyxin B-immobilized fiber column direct hemoperfusion. $p$ value, appropriate group versus delayed group. * Patients who underwent PMX-DHP for at least $2 \mathrm{~h}$ or more were included in the analysis.

$0 \%$ vs. $80 \%, p=0.015$; respectively). The reason for these results is that preparing PMX-DHP equipment immediately after birth is possible in EOS cases, which can be predicted early based on information such as maternal fever and chorioamnionitis. In addition, by cooperating with medical engineers, it is possible to induce PMXDHP immediately after onset. Conversely, in LOS cases, securing blood access takes times because of the difficulty in detecting the onset and the inability of using umbilical arteries and veins.

There are some restrictions on PMX-DHP use for both adult and pediatric patients according to the national health insurance system in Japan. The national health insurance guidelines of PMX-DHP in Japan meet the pediatric systemic inflammatory response syndrome diagnostic criteria [8] for patients younger than 18 years who are suspected of having endotoxemia or Gram-negative bacterial infection. In addition, the recommended continuous duration is $2 \mathrm{~h}$ per session in principle [7], and 2 times is the upper limit for the number of times it should be performed in Japanese clinical settings. Therefore, it may be difficult to systematically extend the total duration time of PMX-DHP in Japan due to these restrictions.

In summary, we believe that earlier PMX-DHP induction should be considered for neonatal septic shock, rather than long-term PMX-DHP administration. In order to achieve early PMX-DHP induction, clinicians should aim to diagnose neonatal septic shock and ensure effective blood access as soon as possible. Finally, to accumulate further evidence for evaluating PMX-DHP for neonates, we hope that PMX-DHP columns with low priming volumes (e.g., Toraymyxin $\left.{ }^{\circledR} 01-\mathrm{R}\right)$ can be applied to micropreemie infants with septic shock around the world in the future.

\section{Acknowledgements}

We thank the Juntendo University Urayasu Hospital Medical Engineering Team for lending their expertise on polymyxin B-immobilized fiber column direct hemoperfusion therapy.

\section{Conflict of Interest Statement}

The authors have no conflicts of interest to declare.

\section{Funding Sources}

The authors did not receive any funding.

\section{Author Contributions}

N.N. designed the study and wrote the manuscript; K.O. and T.S. reviewed the manuscript and supervised the whole study process. All authors read and approved the final manuscript. 


\section{References}

1 Mitaka C, Kusao M, Kawagoe I, Satoh D, Iba $\mathrm{T}$, Ronco C. Impact of extended duration of polymyxin B-immobilized fiber column direct hemoperfusion on hemodynamics, vasoactive substance requirement, and pulmonary oxygenation in patients with sepsis: an observational study. Blood Purif. 2021:1.

2 Nishizaki N, Nakagawa M, Hara S, Oda H, Kantake M, Obinata K, et al. Effect of PMXDHP for sepsis due to ESBL-producing E. coli in an extremely low-birthweight infant. Pediatr Int. 2016 May;58(5):411-4.
3 Nishizaki N, Hirano D, Miyasho T, Obinata K, Shoji H, Shimizu T. Evaluation of urinary IL-6 in neonates with septic shock treated with polymyxin B-immobilized fiber column. Pediatr Int. 2017 Sep;59(9):1032-3.

4 Nishizaki N, Hara T, Obinata K, Nakagawa M, Shimizu T. Clinical effects and outcomes after polymyxin B-immobilized fiber column direct hemoperfusion treatment for septic shock in preterm neonates. Pediatr Crit Care Med. 2020 Feb;21(2):156-63.

5 Nishizaki N. Suggestions and tips regarding polymyxin B-immobilized fiber column direct hemoperfusion of neonates with sepsis. Acute Crit Care. 2020 Aug; 35(3):226-7.
6 Nishizaki N, Shima T, Watanabe A, Obinata K, Shimizu T. Unsatisfactory short-term neurodevelopmental outcomes of preterm infants who received polymyxin B-immobilized fiber column-direct hemoperfusion for septic shock. Tohoku J Exp Med. 2021 Apr;253(4):275-81.

7 Shoji H. Extracorporeal endotoxin removal for the treatment of sepsis: endotoxin adsorption cartridge (Toraymyxin). Ther Apher Dial. 2003 Feb;7(1):108-14.

8 Goldstein B, Giroir B, Randolph A. The members of the international consensus conference on pediatric sepsis. International pediatric sepsis consensus conference: Definitions for sepsis and organ dysfunction in pediatrics. Pediatr Crit Care Med. 2005 Jan;6(1):2-8. 